ISSN 0001-6012/2020/62/3/140-144 Acta Médica Costarricense, (c 2020 Colegio de Médicos y Cirujanos de Costa Rica

\title{
Opinión
}

\section{Logros académicos en Geriatría y Gerontología en Costa Rica}

\section{(Academic achievements in Geriatrics and Gerontology in Costa Rica)}

Fernando Morales-Martínez

\section{Resumen}

El análisis panorámico de la información demográfica en Costa Rica y Latinoamérica evidencia el impacto que tiene y tendrá la demanda de servicios especializados en Geriatría y Gerontología, en los diferentes niveles de atención en un sistema nacional de salud y la comunidad. Esto involucra un enfoque multidisciplinario, interdisciplinario y transdisciplinario, por la complejidad inherente a las situaciones. El desarrollo histórico de la geriatría internacional y nacional, junto con los invaluables aportes académicos mediante diferentes modalidades de enseñanza: educación médica continua, grado y postgrado, con la participación activa del Hospital Nacional de Geriatría y Gerontología de la Caja Costarricense de Seguro Social y de la Facultad de Medicina de la Universidad de Costa Rica, colocan al país en una posición aventajada en esta área de formación de recurso humano; los logros académicos obtenidos en los últimos 28 años permiten un escenario más optimista para las personas adultas mayores en el país.

Descriptores: Geriatría, Gerontología, docencia, demografía

\section{Abstract}

The panoramic analysis of demographic information in Costa Rica and Latin America, demonstrates the impact it has and will have the demand for specialized services in Geriatrics and Gerontology at different levels of care in the national health system and the community; which involves a multidisciplinary, interdisciplinary and transdisciplinary approach due to the complexity of the situations. The historical development of international and national geriatrics, and the invaluable academic contributions in the different teaching modalities: Continuing, Undergraduate and Postgraduate Medical Education, with the active participation of the National Hospital of Geriatrics and Gerontology of the Costa Rican Social Security SYSTEM and of The Faculty of Medicine of the University of Costa Rica, places Costa Rica in an advantageous position in this area of human resource training, the academic achievements obtained in the last 28 years allow us to see a more optimistic outlook for older adults in the country.

Keywords: Geriatrics, Gerontology, Teaching, Demography.

Fecha recibido: 18 de diciembre 2019

Fecha aprobado: 29 de julio 2020

La cantidad de personas mayores de la región de América Latina y el Caribe se incrementará de manera sostenida en las próximas décadas. Según indica la síntesis del documento elaborado por la Comisión Económica para América Latina y el Caribe (CEPAL), en el 2025, 57 millones de personas mayores se sumarán a los 41 millones existentes y, entre 2025 y 2050, ese incremento será de 86 millones de personas. De tal forma, esta población crecerá rápidamente $(3,5 \%)$ y con un impulso mayor que el que muestra la población de menor edad. De hecho, la velocidad de cambio de esta población será entre tres y cinco veces mayor que la de la población total entre 2000 - 2025 y 2025 - 2050, respectivamente. Con el aumento de la longevidad, las personas 
más viejas entre los mayores se incrementarán; la población mayor de 75 años pasará de un $2 \%$ a un $8 \%$ entre 2000 y 2050.

Estos cambios demográficos, tienen y tendrán importantes efectos de muy diversa intensidad que pasan al primer plano de las preocupaciones ciudadanas y políticas, sobre todo por las consecuencias sociales y económicas que pueden tener a largo plazo en la familia, la salud, el mercado laboral, la educación, las políticas de inmigración y los sistemas previsionales. El modelo de atención a personas adultas mayores debe conformarse a partir de la realidad de cada país, de los recursos disponibles y desde un enfoque de envejecimiento y vejez que permita maximizar las acciones, los recursos y las oportunidades, para garantizar una sociedad inclusiva en todas las edades que, al amparo del cambio de la estructura poblacional y del cambio epidemiológico que ello representa, permita alcanzar un envejecimiento y disfrutar la vejez con calidad de vida y con pleno ejercicio de la ciudadanía.

El Estado costarricense debe garantizar la protección integral de las personas adultas mayores ante el proceso acelerado de envejecimiento de su población, asumir el reto en la formulación y ejecución de acciones nacionales en materia de envejecimiento y vejez, adoptar las medidas necesarias en todas las áreas y sectores, y el compromiso de promover y proteger los derechos humanos y las libertades fundamentales de este grupo.

En el plano académico en Geriatría y Gerontología en Costa Rica, lo expuesto obliga a una atención más precisa con un enfoque multidisciplinario, interdisciplinario y transdisciplinario; hacia la prevención y promoción de la salud, para que cada persona asuma la responsabilidad de su autocuidado en salud, en tanto que el sistema contribuye a modificar los condicionantes ambientales, físicas, sociales y culturales; los modelos de atención basados en las personas adultas mayores, familia, la comunidad y la atención primaria; y en este sentido es preciso reconocer el contexto de los logros históricos académicos del país y plantear los retos actuales para la especialidad.

\section{Antecedentes históricos}

Aunque en 1959, en el informe de la Organización Mundial de la Salud (OMS), en el ejecutado 8.2, se enunció lo siguiente: "En los planes de estudio de las facultades de Medicina y Escuela de Enfermería, deberá darse un lugar importante a los problemas del envejecimiento", no fue sino hasta 1975, según informe $\mathrm{N}^{\circ}$ 507, cuando la OMS se pronunció en favor del desarrollo de una rama de la medicina especializada en la atención de los adultos mayores. Al año siguiente se abrió la primera Cátedra de Geriatría en la Universidad de Glasgow, con el doctor Sir Ferguson Anderson, y surge entonces la Geriatría moderna, aplicando la evaluación integral por parte del equipo interdisciplinario, con la idea de considerar al adulto mayor en sus aspectos médicos, psíquicos, funcionales y sociales. En 1964, el Dr. James Williamson, profesor de Geriatría de la Universidad de Edimburgo, describió las necesidades no reportadas en las personas adultas mayores, e identificó las amenazas en la salud, al mismo tiempo que indicó que el bienestar de este grupo poblacional estaba ignorado. Específicamente, documentó problemas de la movilidad, visuales, auditivos, mentales, sociales, de vivienda y financieros, así como cardiopatías, neuropatías, incontinencias, artralgias y osteopatías.

En 1982, convocada por las Naciones Unidas, se realizó la Primera Asamblea Mundial del Envejecimiento. Veinte años después, en 2002, se efectuó la Segunda Asamblea Mundial, donde se sentaron las bases de la formación y ejecución de programas en beneficio de los adultos mayores.

\section{Antecedentes en Costa Rica}

Los médicos docentes de la Universidad de Costa Rica (UCR), en la especialidad médica de Geriatría y Gerontología, se venían formando en el extranjero desde la década de los ochentas del siglo anterior, con el apoyo de la Caja Costarricense de Seguro Social (CCSS), de la Escuela de Medicina de la UCR y de las universidades extranjeras.

\begin{tabular}{|c|c|}
\hline \multicolumn{2}{|c|}{$\begin{array}{l}\text { Cuadro 1. Fundadores, en 1991, de la especialidad en Geriatría y Gerontología, según } \\
\text { área de formación académica, con el Sistema de Estudios de Posgrado (SEP) de la } \\
\text { Universidad de Costa Rica, y el Centro Nacional de Desarrollo Estratégico en Salud y } \\
\text { Seguridad Social (CENDEISSS), de la Caja Costarricense de Seguro Social }\end{array}$} \\
\hline Nombre & Área de formación académica \\
\hline Dr. Fernando Morales Martínez & $\begin{array}{l}\text { Especialista en Geriatría y Gerontología. Especialista en } \\
\text { Administración de Servicios de Salud. Máster en Geriatría y } \\
\text { Gerontología Médica }\end{array}$ \\
\hline Dra. María de los Ángeles Solís Umaña & Especialista en Geriatra y Gerontología \\
\hline Dr. Carlos Luis Alpízar Quesada & $\begin{array}{l}\text { Especialista en Geriatría y Gerontología (primer especialista } \\
\text { inscrito en el Colegio de Médicos y Cirujanos en 1969) }\end{array}$ \\
\hline Dr. Antonio Rodríguez Aragonés & Especialista en Salud Pública \\
\hline Dr. Eduardo Castro Mendoza & Especialista en Gastroenterología \\
\hline Dr. Franz Chaves Chavarría & Especialista en Reumatología \\
\hline Máster Flory Saborío Hernández & Trabajadora social \\
\hline
\end{tabular}


Luego, en el país se logró la conformación del programa de postgrado en la especialidad médica, con los fundadores de la especialidad citados en el Cuadro 1. Fue a partir de 1991, cuando se inició la formación de especialistas a nivel nacional bajo el Sistema de Estudios de Posgrado de la UCR y el Centro Nacional de Desarrollo Estratégico en Salud y Seguridad Social (CENDEISSS) de la CCSS. En un principio este programa tenía una duración de cuatro años: dos años de Medicina Interna dirigida y dos años de Geriatría y Gerontología, sin embargo, a partir de 2005 se amplió un año más de Geriatría y Gerontología, para un total de cinco años.

El postgrado clínico precedió la inclusión de contenidos temáticos en el plan de estudios de la carrera de Medicina y Cirugía en la UCR; luego, por medio de un equipo de trabajo de médicos docentes y el apoyo de la Dirección de la Escuela de Medicina de la UCR, en 1999 se logró la apertura de la Sección Académica de Geriatría de la Escuela de Medicina de la UCR en ese hospital, con los cursos de Fisiopatología y Semiología y Medicina Interna I, además del curso de Geriatría y Gerontología para los estudiantes del Departamento Académico del Hospital México y del Hospital San Juan de Dios.

Un logro más para el país fue que, en 2008, la OMS y la Organización Panamericana de la Salud (OPS) nombraron al Hospital Nacional de Geriatría y Gerontología como Centro Colaborador en Educación en Geriatría y Gerontología a nivel latinoamericano, labor desarrollada en forma continua hasta la fecha, y gracias a la cual 120 profesionales de 14 países latinoamericanos han realizado una pasantía clínica.

Más recientemente, en octubre de 2015, la OMS presentó el Informe Mundial sobre Envejecimiento y Salud, que constituye la hoja de ruta de planes y programas que deben regir a todos los países del mundo, donde claramente está incluida la educación en Geriatría y Gerontología.

\section{Situación actual}

Desde 2014, la antigua Sección Académica de Geriatría de la Escuela de Medicina de la UCR, en el Hospital Nacional de Geriatría y Gerontología, pasó a ser el actual el Departamento Clínico de Geriatría y Gerontología; en 2015 y por medio de la modificación curricular sugerida en el plan de mejora del proceso de reacreditación de la Escuela de Medicina, se aprobaron los cursos ME-0315 Geriatría y Gerontología I (IS) y ME-316 Geriatría y Gerontología II (IIS), ambos de carácter obligatorio en el quinto año de la carrera de Licenciatura en Medicina y Cirugía de la UCR, los cuales han resultado muy bien acogidos por los estudiantes para el desarrollo y ejercicio de su profesión. Para 2019, 3004 médicos graduados de la UCR han realizado rotaciones clínicas en este hospital.

Al reconocer la importancia de la educación médica permanente, desde 1984 se desarrolla anualmente la Semana Nacional e Internacional de Geriatría y Gerontología; han asistido cerca de 5000 profesionales en los últimos 34 años y han participado aproximadamente 70 distinguidos profesores geriatras invitados de las mejores universidades de Europa, Norteamérica y Latinoamérica, así como profesionales nacionales, más recientemente con el apoyo de la Fundación Costarricense para la Docencia e Investigación en Geriatría y Gerontología (FUNDOGEN).

\section{Competencias de formación}

La formación de grado de los estudiantes universitarios, especialmente los de la carrera de medicina, debe tomar en cuenta competencias profesionales generales junto con el

\section{Cuadro 2. Habilidades que deberán dominar en el área de Geriatría y Gerontología, los estudiantes de la carrera de Medicina de la Escuela de Medicina de la Universidad de Costa Rica, 2020}

- Evaluar los aspectos físicos, mentales, sociales y funcionales de la salud de los pacientes de edad avanzada.

- Distinguir el proceso normal del envejecimiento de sus manifestaciones patológicas.

- Dar prioridad a los problemas clínicos y procedimientos diagnósticos y terapéuticos que más impacto tengan sobre la conservación o recuperación de las funciones.

- Elaborar un plan de atención según los problemas identificados, tomando como puntos preferentes de decisión la esperanza de vida, la situación funcional de base y el pronóstico y la calidad de vida futura, utilizando apropiadamente para ello los recursos médicos y paramédicos disponibles en el hospital y en la comunidad.

- Conocer los principios y prácticas del cuidado ambulatorio crónico en los pacientes con enfermedades irremediables y el cuidado de pacientes terminales.

- Conocer la organización y los servicios de apoyo disponibles para el cuidado de los pacientes adultos mayores en el hospital y en la comunidad.

- Conocer los propósitos y funciones de los diferentes miembros del equipo multidisciplinario que participan en el cuidado del paciente.

- Ser capaz de comunicarse con el paciente de edad avanzada.

- Tener una actitud optimista durante la atención del adulto mayor. 
aprendizaje de disciplinas especializadas como la Geriatría, que requiere otras competencias. Por ejemplo, se debe contribuir a mejorar la capacidad de los graduados para elaborar una historia clínica geriátrica y realizar la exploración del estado físico y mental del adulto mayor, así como para evaluar su capacidad funcional y los recursos sociales con los que cuenta. También se deben desarrollar habilidades que les permita utilizar la información obtenida para formular el diagnóstico diferencial, identificar los problemas que presenta el paciente y elaborar un plan inicial para su atención y control. Por último, deberá contribuir a comprender la necesidad de trabajar en equipos multi e interdisciplinarios, para lograr atender de forma adecuada a los adultos mayores.

Los estudiantes de Medicina deberán dominar una serie de habilidades citadas en el Cuadro 2, y así el futuro médico asumirá una actitud positiva hacia los adultos mayores, en general, y hacia los enfermos y las personas frágiles, en particular. Además, deberá caracterizarse por sus sentimientos de solidaridad y por mantener una actitud de servicio, respeto y empatía hacia las personas mayores y su entorno, muy particularmente hacia sus familias, aun ante una enfermedad terminal. También tendrá que desarrollar una elevada capacidad de negociación, tanto para abordar la relación terapéutica como para trabajar en equipo. Deberá conocer, respetar y defender los derechos y los marcos legales relativos a las personas adultas mayores. Asimismo, deberá tener una actitud de liderazgo al estar comprometido con las tareas de prevención, con el desarrollo de modelos de atención y con la

\begin{tabular}{|lc|}
\hline \multicolumn{2}{|c|}{$\begin{array}{c}\text { Cuadro 3. Áreas temáticas investigadas para optar } \\
\text { al título profesional de Especialista en Geriatría y } \\
\text { Gerontología, 1993 - 2018 }\end{array}$} \\
\hline \multicolumn{1}{|c|}{ Áreas temáticas investigadas } & $\begin{array}{c}\text { Número de } \\
\text { publicaciones }\end{array}$ \\
\hline Nutrición & 14 \\
Patología psiquiátrica & 12 \\
Epidemiología & 10 \\
Atención comunitaria & 9 \\
Patología infecciosa & 8 \\
Rehabilitación funcional & 7 \\
Cardiopatías & 6 \\
Neuropatías & 6 \\
Neumopatías & 5 \\
Tratamiento farmacológico & 5 \\
Uropatías & 5 \\
Sangrado digestivo & 3 \\
Otras temáticas & 30 \\
\hline $\begin{array}{l}\text { Fuente: elaboración propia a partir de Registro de trabajos de graduación de } \\
\text { especialidad. Departamento Clíico de Geriatría y Gerontología de la Escuela de } \\
\text { Medicina / Sistema de Estudios de Posgrado, Universidad de Costa Rica, Costa Rica, } \\
\text { 2018 }\end{array}$ \\
\hline
\end{tabular}

conducción de equipos de salud multidisciplinarios dedicados a los adultos mayores.

En el marco del postgrado, se han formado 142 médicos especialistas, hay 60 médicos residentes en formación y en el futuro se necesitará una cantidad aún mayor.

\section{Investigación clínica}

Siempre en el ámbito universitario, la docencia en conjunto con la investigación clínica que realizan los médicos residentes y los especialistas docentes, sirve de complemento al trabajo de acción social.

El programa de postgrado incluye, como requisito de graduación para obtener el título de especialista, la elaboración y defensa pública de un trabajo de investigación clínica; además, las investigaciones clínicas y su consecuente publicación confieren puntos para ascenso en régimen académico de los docentes universitarios. Se han efectuado más de un centenar de trabajos de investigación, de gran importancia país (cuyas áreas temáticas se presentan en el en el Cuadro 3), tal es el caso del estudio con la población centenaria de Nicoya, Guanacaste, en 2017.

\section{Retos}

Queda claro que Costa Rica tiene una posición aventajada en esta área de formación de recurso humano; se ha venido preparando paulatina y sistemáticamente para enfrentar la gran demanda de servicios especializados de una manera oportuna y eficaz, mediante los logros académicos obtenidos en los últimos 28 años, los cuales permiten ver un panorama más optimista para la población adulta mayor. Desde su creación, el Departamento Clínico de Geriatría y Gerontología ha crecido hasta contar con 35 docentes activos (32 de ellos médicos especialistas en Geriatría y Gerontología), todos comprometidos con el espíritu de trabajo en equipo interdisciplinario, tan necesario para continuar con el fortalecimiento de la formación del recurso humano y dar respuesta a las demandas actuales y futuras, que permitan atender eficaz y eficientemente el proceso de envejecimiento de la población.

En la actualidad, Costa Rica tiene un médico especialista en Geriatría y Gerontología por cada 4500 personas adultas mayores; es el único país latinoamericano que obtiene este logro académico y profesional; sin embargo, la meta país es llegar a un médico geriatra por cada 1000 personas adultas mayores.

Para una atención integral es necesario el establecimiento de programas precisos, diseñados para solventar las necesidades de la población adulta mayor desde el ámbito de la salud y de los servicios de apoyo comunitario. Con este criterio como base, se propone un modelo de atención integral y progresiva, dirigido a reincorporar a las personas mayores a su medio 
habitual, con definición de una red de recursos institucionales desde el ámbito especializado, mediante los niveles de atención geriátrica desarrollados en Costa Rica y una red social que parte de la comunidad con el apoyo estatal.

Agradecimientos: A la Dra. Grettchen Flores Sandí, docente catedrática y directora del Departamento Clínico de Medicina Legal, Escuela de Medicina, UCR; a la Dra. Faridy Helo Guzmán, docente del Departamento Clínico de Geriatría y Gerontología, Escuela de Medicina, UCR, y a la Sra. Sonia Zeledón Mora, secretaria del Departamento Clínico.

\section{Lecturas recomendadas:}

1. Comisión Económica para América Latina y el Caribe (CEPAL). Hacia un cambio de paradigma sobre el envejecimiento y la vejez. Santiago, Chile: CEPAL; 2010.

2. Consejo Nacional de la Persona Adulta Mayor (CONAPAM), Universidad de Costa Rica (UCR). I Informe Estado de situación de la persona adulta mayor en Costa Rica. San José, Costa Rica: UCR, CONAPAM; 2008.
3. Consejo Nacional de la Persona Adulta Mayor (CONAPAM). Red de Atención Progresiva para el Cuido Integral de las Personas Adultas Mayores en Costa Rica. San José, Costa Rica: CONAPAM; 2010.

4. Cano C, Gutiérrez L, Marín P, Martínez F, Peláez M, Rodríguez L, et al. Propuesta de contenidos mínimos para los programas docentes de pregrado en Medicina Geriátrica en América Latina. Rev Panam Salud Pública/Pan Am J Public Health 2005; 17: 429-437.

5. Organización Mundial de la Salud (OMS). Informe mundial sobre el envejecimiento y la salud. Ginebra, Suiza: OMS 2015.

6. Michel JP, Bong H. Filling the geriatric education gap around the world. JAMDA. 2015; 16: 1010-1013.

7. Philp I. The contribution of geriatrics medicine to integrated care for older people. Age Ageing. 2015; 44: 11-15.

8. Morley JE. A brief history of geriatrics. J Gerontol A boil Sci Med Sci. 2004; 59: $1132-1152$.

9. Morales F. Is Geriatric Medicine possible in a Midde Income Country? The case of Costa Rica. JAGS. 2017; 65:1870-1875.

10. Morales F. Geriatric Medicine Bridges: Scotland - Costa Rica. J R Coll Physicians Edinb. 2017; 47: 324-330.

11. Morales F. A teaching Geriatric Hospital. J Nutr Health Aging. 2018; 22: 1-5.

12. Registro de trabajos de graduación de especialidad. Departamento Clínico de Geriatría y Gerontología de la Escuela de Medicina / Sistema de Estudios de Posgrado, Universidad de Costa Rica. Costa Rica; 2018. 